\title{
Use of a Modification of the Patel-Teja Equation of State + van der Waals-Platteeuw Theory Based Model for Predicting Hydrate Phase Boundary of Methane - Ethylene Glycol - Water System from Ice Point Data of Aqueous Solution
}

\author{
A.H. Mohammadi and D. Richon \\ Mines Paris, ParisTech, CEP/TEP. CNRS FRE 2861, 35 rue Saint Honoré, 77305 Fontainebleau - France \\ e-mail: amir-hossein.mohammadi@ensmp.fr -dominique.richon@ensmp.fr
}

\begin{abstract}
Résumé - Utilisation de l'équation de Patel-Teja associée au modèle de van der Waals-Platteeuw pour estimer les équilibres de phase d'hydrates de méthane dans des systèmes eau-éthylène glycol à partir de points de congélation de solutions aqueuses - Un souci important avec les canalisations de pétrole/gaz et la production/les unités de traitement est la possibilité de restriction et de colmatage des écoulement dus à la formation d'hydrate de gaz. En effet le résultat étant de sérieux problèmes opérationnels, économiques et de sécurité. Des inhibiteurs organiques tels que le méthanol et l'éthylène-glycol sont couramment employés pour prévenir la formation d'hydrates de gaz. La connaissance précise des équilibres de phases de solutions comprenant des hydrates en présence d'inhibiteurs est donc cruciale afin d'être en mesure d'éviter tout problème de formation d'hydrate de gaz et pour concevoir et optimiser la production, le transport et les équipements de traitement. Des données expérimentales sont nécessaires pour développer les modèles capables de prévoir le comportement de phases en présence d'hydrates. Les mesures d'équilibre de phases comportant des hydrates en présence d'inhibiteurs (éthylène glycol) se révèlent beaucoup plus difficiles que celles du point de congélation des solutions concernées, et ce en particulier, aux fortes concentrations d'inhibiteur. Ceci est partiellement dû au fait que les inhibiteurs décalent des frontières de formation d'hydrate des fluides pétroliers vers les hautes pressions, d'où de plus grandes difficultés expérimentales concernant les mesures d'équilibres de phase. Dans ce travail, nous examinons les besoins de données d'équilibre de phase avec hydrate et inhibiteurs pour ajuster des paramètres de modèles thermodynamiques basés sur l'utilisation d'une modification de l'équation de Patel-Teja associé au modèle de van der Waals-Platteeuw. Nous considérons deux voies d'ajustement : utilisation de données d'équilibres, utilisation de points de congélation. Les résultats de notre étude prouvent que l'emploi de valeurs de points de congélation suffit pour l'ajustement de paramètres d'un modèle thermodynamique tout en permettant des prévisions acceptables des équilibres de phase d'hydrate en présence d'inhibiteur. En conséquence on peut conclure que les données expérimentales d'équilibre de phase en présence d'hydrates ne sont indispensables dans la mesure où l'on dispose de points de congélations.
\end{abstract}




\begin{abstract}
Use of a modification of the Patel-Teja equation of state + van der Waals-Platteeuw theory based model for predicting hydrate phase boundary of methane-ethylene glycol-water system from ice point data of aqueous solution - A major concern with the oil/gas pipelines and production/processing facilities is the possibility of flow restriction and blockage due to gas hydrate formation, which can lead to serious operational, economic and safety problems. Organic inhibitors such as methanol and ethylene glycol are normally used for preventing gas hydrate formation. Accurate knowledge of hydrate phase equilibrium in the presence of inhibitors is therefore crucial to avoid gas hydrate formation problems and to design/optimize production, transportation and processing facilities. Experimental data are needed for developing models capable of predicting hydrate phase behavior. In general, measuring hydrate phase equilibrium in the presence of inhibitor is more difficult than measuring ice point of inhibitor aqueous solution, particularly at high concentrations of inhibitor. This is partly due to the fact that the presence of inhibitor shifts hydrate phase boundaries to high pressures, which leads to difficulties in hydrate phase equilibria measurements. In this work, we examine the need for hydrate phase equilibrium data in the presence of inhibitor (ethylene glycol) aqueous solutions for tuning parameters of a thermodynamic model based on a modification of the Patel-Teja equation of state $+v a n$ der Waals-Platteeuw theory. We consider two cases for tuning: use of ice point data or, using hydrate phase equilibrium data. The results show that using only ice point data of inhibitor aqueous solution for tuning thermodynamic model can lead to acceptable predictions of hydrate phase equilibrium in the presence of inhibitor and therefore experimental hydrate phase equilibrium data are not strictly indispensable.
\end{abstract}

\section{INTRODUCTION}

Gas hydrates are solid crystalline compounds formed from mixtures of water and guest molecules of suitable sizes under low-temperatures and high-pressures. In the hydrate lattice, water molecules form hydrogen-bonded cagelike structures, encapsulating the guest molecules, which generally consist of low molecular diameter gases and organic compounds [1]. Conditions of high-pressure and low-temperature as well as presence of water and hydrocarbon leading to gas hydrate formation may be found in oil and gas production, transportation and processing facilities. Formation of gas hydrates can lead to serious operational, economic and safety problems in petroleum industry due to potential blockage of oil and gas equipment [1]. Organic inhibitors, such as methanol and ethylene glycol are normally used to inhibit gas hydrate formation [1]. A good knowledge of the hydrate phase behavior in the presence of inhibitors is therefore essential for safe and economical design and operation of associated fields, pipelines and production/processing facilities [1]. Experimental data are needed for successfully developing and validating models capable of predicting hydrate phase behavior. Gas hydrate dissociation data in the presence of inhibitors are normally used to adjust models parameters. In general, experimental measurements of hydrate phase equilibria are often expensive and time consuming, and if not performed carefully, can be highly inaccurate $[1,2]$. The consequences of inaccuracy, particularly in petroleum exploration and production operations, can be costly [2]. Inaccurate experimental measurements of hydrate phase boundaries could lead to the over-estimation/under-estimation of inhibitor capabilities, resulting in inadvertent operations within the hydrate stability zone and overlunder-inhibition and extra costs [2]. Fortunately, measuring ice point of aqueous solutions is easier than measuring hydrate phase equilibria, particularly at high concentrations of inhibitors in aqueous solutions. This is partly due to the fact that the presence of inhibitors, especially high concentrations of inhibitors, shift hydrate phase boundaries to high pressures, which can lead to difficulties in hydrate phase equilibria measurements.

The aim of the present work is to examine the possibility of using ice point data of inhibitor (e.g., ethylene glycol) aqueous solutions for tuning parameters of a thermodynamic model in order to reliably predict hydrate phase equilibria in the presence of inhibitor. A thermodynamic model [3] based on combination of the Valderrama modification of the PatelTeja equation of state (VPT-EoS) [4] with non-density dependent mixing rules (NDD) [5] is used for modeling the phase behavior of solutions containing organic inhibitors. The VPT-EoS [4] with NDD [5] is also used to model the gas phase. The ice phase is modeled by correcting the vapor pressure of ice using the Poynting correction [6]. The hydrate phase is modeled using the solid solution theory of van der Waals and Platteeuw [7]. The performance of the model is evaluated using some selected hydrate dissociation data and ice point data from the literature. It is shown that using only experimental data on ice point of inhibitor aqueous solution for tuning thermodynamic model can lead to acceptable predictions of hydrate stability zone, thereby eliminating the need to measure the hydrate phase equilibria in the presence of inhibitor aqueous solution. 


\section{THERMODYNAMIC MODEL}

The model used in this work is originally based on a comprehensive thermodynamic model, the Heriot-Watt University Hydrate Model (HWHYD model) [3], which is capable of predicting different scenarios in water-hydrocarbon systems and hydrate phase equilibrium.

\subsection{Equation of State}

The VPT-EoS [4] is used for modeling fluid phases, as it is believed that this EoS is a strong tool for modeling systems containing water, alcohols and glycols [5]. In this equation of state, the alpha function, $\alpha\left(T_{r}\right)$, is given using the following equation [4]:

$$
\alpha\left(T_{r}\right)=\left[1+F\left(1-T_{r}^{\Psi}\right)\right]^{2}
$$

where $T$ is the temperature, and $\Psi=0.5$. The subscripts $c$ and $r$ denotes reduced property, respectively. The coefficient $F$ is given by [4]:

$$
F=0.46286+3.58230\left(\omega Z_{c}\right)+8.19417\left(\omega Z_{c}\right)^{2}
$$

where $Z_{c}$ is the critical compressibility factor, and $\omega$ is the acentric factor. Avlonitis et al. [5] relaxed the constraints on $F$ and $\Psi$ for water and methanol in order to improve the predicted vapor pressure and saturated volume for these compounds [5]:

$$
\begin{array}{lll}
\text { Methanol: } & F=0.76757, & \Psi=0.67933 \\
\text { Water: } & F=0.72318, & \Psi=0.52084
\end{array}
$$

Later, Tohidi-Kalorazi [6] relaxed the alpha function for water, $\alpha_{w}\left(T_{r}\right)$, using experimental water vapor pressure data in the range of 258.15 to $374.15 \mathrm{~K}$, in order to improve the predicted water fugacity [6]:

$$
\alpha_{w}\left(T_{r}\right)=2.4968-3.0661 T_{r}+2.7048 T_{r}^{2}-1.2219 T_{r}^{3}
$$

The relation of Tohidi-Kalorazi [6] for water is used in the present work.

Glycols can be modeled using the original VPT EoS [4]. In order to obtain satisfactory results for these components, it was not found necessary to adopt approaches similar to that for water and methanol [6].

\subsection{Mixing Rules}

Nonpolar-nonpolar binary interactions in fluid mixtures are described by applying classical mixing rules as follows [5]:

$$
a=\sum_{i} \sum_{j} x_{i} x_{j} a_{i j}
$$

$$
\begin{gathered}
b=\sum_{i} x_{i} b_{i} \\
c=\sum_{i} x_{i} c_{i} \\
a_{i j}=\left(1-k_{i j}\right) \sqrt{a_{i} a_{j}}
\end{gathered}
$$

where $a, b$ and $c$ are attractive term, repulsive term and third parameter of the EoS, respectively and $k_{i j}$ is the binary interaction parameter (BIP) in classical term. $x$ stands for mole fraction of component.

For polar-nonpolar interaction, however, the classical mixing rules are not satisfactory and therefore more complicated mixing rules are necessary. In this work, the NDD mixing rules developed by Avlonitis et al. [5] are applied to describe mixing in the $a$-parameter [5]:

$$
a=a^{C}+a^{A}
$$

where $a^{C}$ is given by the classical quadratic mixing rules (Equations 4,7 ). The term $a^{A}$ corrects for asymmetric interaction which cannot be efficiently accounted for by classical mixing [5]:

$$
\begin{gathered}
a^{A}=\sum_{p} x_{p}^{2} \sum_{i} x_{i} a_{p i} l_{p i} \\
a_{p i}=\sqrt{a_{p} a_{i}} \\
l_{p i}=l_{p i}^{0}-l_{p i}^{1}\left(T-T_{0}\right)
\end{gathered}
$$

where $p$ is the index of polar components. $T_{0}$ stands for the reference temperature $(273.15 \mathrm{~K}) . l_{p i}^{0}$ and $l_{p i}^{1}$ are the binary interaction parameters in asymmetric term.

Using the above EoS [4] and the associated mixing rules, the fugacity of each component in fluid phases, $f_{i}$, is calculated from $[5,6]$ :

$$
f_{i}=x_{i} \phi_{i} P
$$

where $P$ is the pressure, and $x_{i}$ and $\phi_{i}$ are the mole fraction and the fugacity coefficient of component $i$, respectively.

\subsection{Ice Phase}

The fugacity of a pure solid can, as for a supersaturated pure liquid, be calculated using the Poynting correction, i.e., assuming that the volume of the supersaturated phase is constant at the volume for the saturated phase $[8,9]$. For ice the expression becomes [6]:

$$
f_{w}^{I}=\phi_{w}^{s a t} P_{I}^{s a t} \exp \left(\frac{v_{I}\left(P-P_{I}^{s a t}\right)}{R T}\right)
$$


where $f_{w}^{I}$ is the fugacity of water in the ice phase, $\phi_{w}^{s a t}$ is the water fugacity coefficient in the vapor phase at pressure equal to the ice vapor pressure, $P_{I}^{s a t}$ is the ice vapor pressure [6], $v_{I}$ is the ice molar volume [6], $R$ is the universal gas constant, and $P$ is the system pressure.

\subsection{Hydrate Phase}

The fugacity of water in the hydrate phase, $f_{w}^{H}$, is given by [9]:

$$
f_{w}^{H}=f_{w}^{\beta} \exp \left(-\frac{\Delta \mu_{w}^{\beta-H}}{R T}\right)
$$

where $f_{w}^{\beta}$ is the fugacity of water in the empty hydrate lattice, which can be calculated using the method reported by Anderson and Prausnitz [9]. $\Delta \mu_{w}^{\beta-H}$ is the chemical potential difference of water between the empty hydrate lattice, $\mu_{w}^{\beta}$, and the hydrate phase, $\mu_{w}^{H}$, which is obtained from the van der Waals and Platteeuw expression [7,9]:

$$
\Delta \mu_{w}^{\beta-H}=\mu_{w}^{\beta}-\mu_{w}^{H}=R T \sum_{m} v_{m} \ln \left(1+\sum_{j} C_{j m} f_{j}\right)
$$

where $v_{m}$ is the number of cavities of type $m$ per water molecule in the unit cell [1], $f_{j}$ is the fugacity of the gas component $j . C_{j m}$ is the Langmuir constant, which accounts for the gas-water interaction in the cavity $[6,10]$.

\section{RESULTS AND DISCUSSIONS}

We study hydrate phase equilibria of methane (the main component of petroleum reservoir fluids) + ethylene glycol (typical organic inhibitor, which is used to inhibit formation of gas hydrates) + water system, as similar results are expected for other systems (for example, systems containing methanol). The critical temperature $\left(T_{\mathrm{C}}\right)$, critical pressure $\left(P_{\mathrm{C}}\right)$, critical volume $\left(v_{C}\right)$ and acentric factor $(\omega)$, of ethylene glycol, water and methane are provided in Table 1 [3]. Table 2 shows the binary interaction parameters between methane-water and methane-ethylene glycol, which were already tuned using experimental data on gas solubility in liquid phase [3].

TABLE 1

Critical Properties and Acentric Factors [3]

\begin{tabular}{c|c|c|c|c}
\hline Compound & $P_{c} / \mathrm{MPa}$ & $T_{c} / \mathrm{K}$ & $v_{c} / \mathrm{m}^{3} \cdot \mathrm{kmol}^{-1}$ & $\omega$ \\
\hline \hline Ethylene glycol & 7.600 & 719.70 & 0.191 & 0.4868 \\
Water & 22.048 & 647.30 & 0.056 & 0.3442 \\
Methane & 4.604 & 190.58 & 0.092 & 0.0108 \\
\hline
\end{tabular}

TABLE 2

BIPs for the VPT-EoS [4] and NDD Mixing Rules [5] $i$ : Water/Ethylene glycol, $g$ : Gas

\begin{tabular}{c|c|c|c}
\hline System & $k_{i-g}=k_{g-i}$ & $l_{i-g}^{0}$ & $l_{i-g}^{1} \times 10^{4}$ \\
\hline Methane-Water & 0.5044 & 1.8302 & 51.72 \\
Methane-Ethylene glycol & 0.3762 & 0.6614 & 22.15 \\
\hline
\end{tabular}

$k_{i-g} \quad$ BIP for the classical mixing rules [5].

$l_{i-g}^{0}$ and $l_{i-g}^{1}$ Constants for the BIP for the asymmetric term $\left(l_{g-i}{ }^{0}=0\right.$ and $\left.l_{g-i}{ }^{1}=0\right)[5]$.

For tuning the BIPs between ethylene glycol and water, two cases are considered. They are:

- only ice point data are used for tuning the BIPs using the objective function (FOB) displayed in Equation 16,

- only hydrate dissociation data are used for tuning the BIPs using the objective function shown in Equation 17.

Objective function 1:

$$
F O B_{1}=\frac{1}{N} \sum_{1}^{N}\left|\frac{T_{\text {Ice,exp }}-T_{\text {Ice, } \text { cal }}}{T_{\text {Ice, } \exp }}\right|
$$

Objective function 2:

$$
F O B_{2}=\frac{1}{M} \sum_{1}^{M}\left|\frac{T_{H y d, \exp }-T_{H y d, c a l}}{T_{H y d, \exp }}\right|
$$

where $N$ and $M$ are the number of ice point data and hydrate dissociation data, respectively. $T_{\text {Ice,exp }}$ and $T_{H y d \text {,exp }}$ stand for the experimental ice point of the aqueous phase and the experimental hydrate dissociation temperature in the presence of inhibitor, respectively. $T_{\text {Ice,cal }}$ and $T_{H y d, c a l}$ represent the calculated ice point of the aqueous phase and the calculated hydrate dissociation temperature in the presence of inhibitor, respectively. In the present work, ice point data reported in CRC Handbook of Chemistry and Physics [11] and hydrate dissociation data of methane in the presence of ethylene glycol aqueous solutions from the literature [12] are used.

Table 3 reports the optimized BIPs values for the ethylene glycol-water system based on the objective functions displayed in Equations 16 and 17. Although the values of the BIPs depend on different factors, such as the number, accuracy and measurement conditions of experimental data as well as appropriate initial guesses for tuning BIPs [13] but our study shows that using the above objective functions leads to approximately the same values of the BIPs. Figure 1 shows a comparison between some ice point data of ethylene glycol aqueous solutions reported in CRC Handbook of Chemistry and Physics [11] and the model results using the BIPs obtained based on Equations 16 and 17. As can be seen, the predictions show satisfactory agreements. Comparisons 
of the results of the model [3] with the experimental data on hydrate phase boundaries of methane in the presence of ethylene glycol aqueous solutions reported in the literature [12] are shown in Figure 2. As can be seen, the experimental data and the predictions of the model [3] on hydrate phase boundaries based on the BIPs obtained using the objective functions indicated in Equations 16 and 17 are in acceptable agreements. The deviations of the model predictions from the experimental data at 50 wt.\% ethylene glycol aqueous solution can be attributed to unreliability of experimental data, as a continues heating method was used to measure hydrate dissociation points rather than step-heating method (or equilibrium method), which is known that this method can lead to uncertainties in the measured data, especially in systems with high volumes of fluids and high concentrations of inhibitors [2].

\section{TABLE 3}

BIPs for the VPT-EoS [4] and NDD Mixing Rules [5].

$i$ : Water, $j$ : Ethylene glycol

\begin{tabular}{c|c|c|c|c|c|c}
\hline System & $\begin{array}{c}\text { Objective } \\
\text { function }\end{array}$ & $k_{i-j}=k_{j-i}$ & $l_{i-j}^{0}$ & $l_{j-i}{ }^{0}$ & $l_{i-j}{ }^{1} \times 10^{4}$ & $l_{j-i}{ }^{1} \times 10^{4}$ \\
\hline $\begin{array}{c}\text { Water- } \\
\text { Ethylene } \\
\text { Glycol }\end{array}$ & Equation 16 & -0.0965 & -0.004 & -0.0092 & 3.54 & 3.08 \\
\cline { 2 - 6 } & Equation 17 & -0.0963 & -0.004 & -0.0090 & 3.54 & 3.10 \\
\hline
\end{tabular}

The reason that the use of ice point data or the data on gas hydrate phase equilibrium for tuning the model can lead to similar predictions of hydrate phase boundaries in the presence of inhibitor aqueous solutions is that both ice and gas hydrate phases equilibria are governed by the fugacity of water in the aqueous phase. An "experimental" water fugacity can be found from both types of equilibria. If the pressure effect is ignored, these "experimental" fugacities can be presented as a function of temperature and composition. The point that should be made is that both types of "fugacities" can be fitted by a single function. Thus, the type of model becomes irrelevant. However, it should be mentioned that this tuning method should not be used for thermodynamic inhibitors that take part in hydrate formation (e.g., 2-propanol) [14].

\section{CONCLUSION}

A thermodynamic model based on a modification of the Patel-Teja equation of state [4] and non-density dependent mixing rules [5] + van der Waals-Platteeuw theory [7] with various databases for tuning (ice point data and hydrate phase equilibrium data) was used to model hydrate phase equilibrium of methane (the main component of petroleum reservoir fluids) in the presence of inhibitor (ethylene glycol, a typical gas hydrate inhibitor) aqueous solution. It was shown that

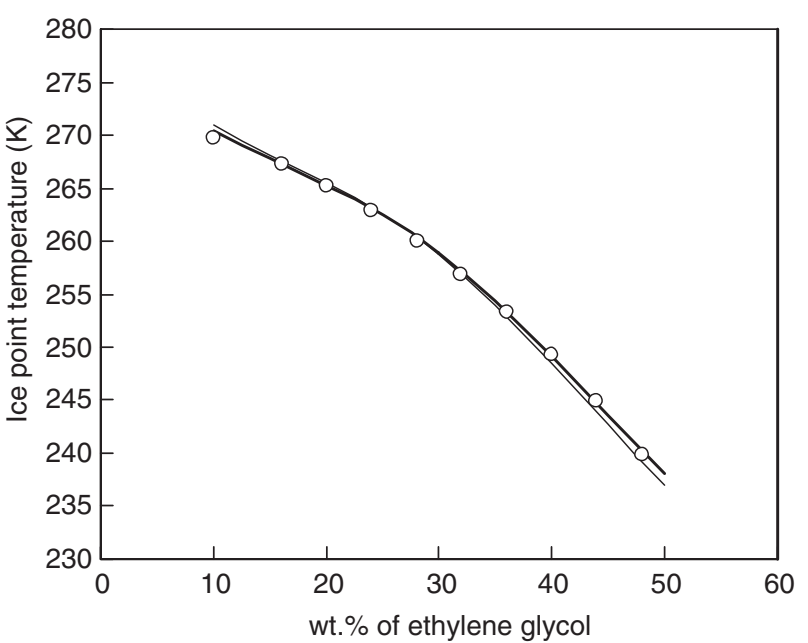

Figure 1

Ice point temperature of ethylene glycol aqueous solution versus wt.\% of ethylene glycol. Solid curve: Model predictions using BIPs obtained based on Equation 17; Bold solid curve: Model predictions using BIPs obtained based on Equation 16; Points: Ice point data reported in CRC Handbook of Chemistry and Physics [11].

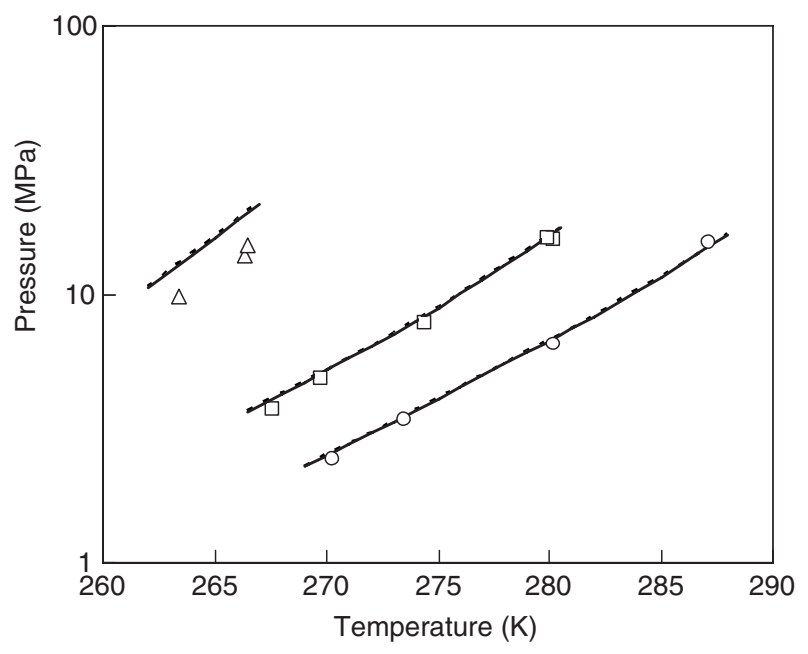

Figure 2

Hydrate phase boundary of methane in the presence of ethylene glycol aqueous solutions. Experimental data [12]: ○: 10 wt.\% ethylene glycol; $\square: 30$ wt.\% ethylene glycol; $\Delta$ : 50 wt.\% ethylene glycol; Dashed curves: Model predictions using BIPs obtained based on Equation 17; Solid curves: Model predictions using BIPs obtained based on Equation 16. 
using data on ice point of inhibitor aqueous solution for tuning thermodynamic model can lead to acceptable predictions of hydrate phase equilibrium in the presence of inhibitor. This is a useful remark to reduce experimental information required to evaluate hydrate stability zone in the presence of inhibitor aqueous solution, where measuring ice point of inhibitor aqueous phase is much easier than measuring gas hydrate inhibition characteristics of inhibitors.

\section{REFERENCES}

1 Sloan E.D. (1998) Clathrate Hydrates of Natural Gases, 2nd ed., Marcel Dekker Inc., New York.

2 Burgass R.W., Tohidi B., Danesh A., Todd A.C. (2002) Application of Quartz Crystal Microbalance to Gas Hydrate Stability Zone Measurements, Proceedings of the Fourth International Conference on Gas Hydrates, Yokohama, Japan, 19-23 May.

3 Heriot-Watt University Hydrate model: http://www.pet.hw.ac.uk/research/hydrate/

4 Valderrama J.O. (1990) A generalized Patel-Teja equation of state for polar and non-polar fluids and their mixtures, J. Chem. Eng. Jpn 23, 87-91.

5 Avlonitis D., Danesh A., Todd A.C. (1994) Prediction of VL and VLL equilibria of mixtures containing petroleum reservoir fluids and methanol with a cubic EoS, Fluid Phase Equilibr. 94, 181-216.
6 Tohidi-Kalorazi B. (1995) Gas Hydrate Equilibria in the Presence of Electrolyte Solutions, PhD Thesis, Department of Petroleum Engineering, Heriot-Watt University, Edinburgh, United Kingdom.

7 Van der Waals J.H., Platteeuw J.C. (1959) Clathrate Solutions, Adv. Chem. Phys. 2, 1-57.

8 Smith J.M., Van Ness H.C. (1987) Introduction to Chemical Engineering Thermodynamics, 4th ed., McGraw-Hill Inc., New York.

9 Anderson F.E., Prausnitz J.M. (1986) Inhibition of Gas Hydrates by Methanol, AIChE J.32, 1321-1333.

10 Kihara T. (1953) Virial Coefficients and Models of Molecules in Gases, Rev. Mod. Phys. 25, 831-843.

11 Weast R.C., Astle M.J., Beyer W.H. (1984-1985) CRC Handbook of Chemistry and Physics, 65th ed., CRC Press Inc., Boca Raton, FL, USA.

12 Robinson D.B., Ng H.J. (1986) J. Can. Petrol. Tech. 26, JulyAugust (Quoted in reference 1).

13 Mohammadi A.H., Chapoy A., Tohidi B., Richon D. (2006) Gas Solubility: A Key to Estimating the Water Content of Natural Gases, Ind. Eng. Chem. Res. 45, 4825-4829.

14 Østergaard K.K., Tohidi B., Anderson R., Todd A.C., Danesh A. (2002) Can 2-Propanol Form Clathrate Hydrates? Ind. Eng. Chem. Res. 41, 2064-2068.

Final manuscript received in May 2007 